\title{
Improving the efficiency and reliability of RPA systems of digital step-down substations and digital grids
}

\author{
Alexey V. Mokeev ${ }^{1,2^{*}}$, Sergey A. Piskunov², Dmitry N. Ulyanov ${ }^{2}$, Evgeniy I. Khromtsov¹,2 \\ ${ }^{1}$ Northern (Arctic) Federal University, Electroenergetics and Electrotechnics Department, Severnaya Dvina emb.17, Arkhangelsk, Russia \\ ${ }^{2}$ Engineering Centre "Energoservice", Kotlasskaya st. 26, Arkhangelsk, Russia
}

\begin{abstract}
The article discusses examples of synchrophasor measurement (SPM) technology application for implementation of inherently selective protection for $35-220 \mathrm{kV}$ step-down substations and $6-20 \mathrm{kV}$ distribution points.
\end{abstract}

\section{Introduction}

One of the most important trends in automation of substations and grids is associated with the integration of digital substation and SPM technologies [1]. The main advantages of using SPM are associated with the reduction of requirements for the amount of data transmitted. There are only six current and voltage synchrophasors required to transfer to calculate dozens of mode parameters for a single feeder on any level of control and protection. On the basis of individual feeder synchrophasors, the parameters of the equivalent power system circuit can be estimated. This opens up new opportunities for building wide area protection systems (WAPS) for substations and electrical grids.

\section{Architecture of RPA systems of step- down substations and distribution points}

Integration of digital substation and SPM technologies will improve the main characteristics of relay protection and automation, primarily related to speed, sensitivity and operational reliability.

The following basic prerequisites for the integration of these technologies should be highlighted:

- development of modern microprocessor technology, which makes it possible to implement more efficient relay protection algorithms;

- the use of modern current and voltage sensors without saturation, residual magnetization, ferroresonance errors;

- the presence of horizontal connections between relay protection and automation devices allows the exchange of fast discrete and analog messages and greatly simplifies the implementation of inherently selective protection;

- identification of the power system equivalent circuit parameters on the basis of the SPM data allows to significantly increase the sensitivity of current protection;

- the use of multifunctional devices with the support of SPM at various levels of substation control will allow the implementation of distributed relay protection and automation systems with effective redundancy even in the event of partial or complete degradation of the communication network at the substation.

At present, most microprocessor devices of the relay protection and automation of the medium voltage facilities include a traditional set of protections, primarily overcurrent protections. Distance and differential protection are used much less. The use of SPM will expand inherently selective protection area based on current or distance principles. The use of additional criteria for fault recognition [2-4] based on synchrophasors will increase the speed and sensitivity of relay protection devices.

Despite the presence of programmable logic and built-in real time clock, logical operations in relay protection and automation are still performed without reference to time. To improve relay protection and automation for various functional purposes, it is promising to use temporal logic, which is used to describe the sequences of events for their interrelation along the time scale [5].

Temporal logic is used in voltage regulation devices in power systems [6]. The use of time logic in protection and automation devices will increase the efficiency of relay protection and automation devices in the implementation of operational interlocks.

More than 50 parameters of the feeder mode can be calculated at any level of control and protection based on 6 current and voltage synchrophasors. When an additional 6 more equivalent synchrophasors are used, about 200 mode parameters can be calculated [7]. When the equivalent synchrophasors are used, it becomes possible to calculate the parameters by symmetrical components, taking into account the influence of harmonics. On the

\footnotetext{
*Corresponding author: a.mokeev@,narfu.ru
} 
basis of SPM data, it becomes possible to evaluate equivalent circuit parameters of the electrical grid, including the power system equivalent parameters. In addition, it becomes possible to recognize modes, including those associated with the processes of starting and self-starting of electric motors.

The integration of digital substation and SPM technologies opens up new opportunities for building distributed multi-level protection and automation systems for substations and electrical grids. Distributed RPA systems will allow wider use of inherently selective protection with the implementation of known or new principles. Actual issues for the creation of these systems are the implementation of measurements of current and voltage synchrophasors with the required accuracy and speed in analog signal converters (ASC), intelligent switching equipment and local RPA devices. At the same time, there is no need to calculate synchrophasors in each subscriber device, which sharply reduces the required level of the computing power of centralized relay protection devices.

The distributed substation RPA system, presented in the report, is based on the use of a combined process and substation bus using synchrophasors instead of sampled values $(\mathrm{SV})$ of currents and voltages, as well as replacing a significant part of MMS messages. This will reduce the traffic of data transmission and provide an increase in the rate of transmission of synchrophasors. Another important task is to increase the speed of relay protection devices with the support of SPM [8].

The use of synchrophasor and GOOSE messaging between substation protections will allow distributed data processing between the protections of individual feeders and implement inherently selective protections. For example, in a relay protection device for substation inputs, it is possible to implement busbar inherently selective protection by using information about the current synchrophasors of medium voltage feeders and voltage synchrophasors on the substation buses. The use of the differential current and additional information about the current synchrophasors of the feeder will increase the speed and sensitivity of busbar protection [8].

Fig. 1 shows the architecture of a distributed relay protection system for a step-down substation (distribution point). The basis of medium voltage switchgear automation is made up of digital switchgear using digital combined current and voltage sensors (DCCVS), smart circuit breakers (SCB) with built-in backup protection, digital position sensors, temperature, arc protection sensors etc. All the listed components inside the switchgear, as well as the relay protection device, are interconnected via redundant low-level deterministic process bus.

Digital combined current and voltage sensors are not only a source of information for IEDs of various functional purposes, but are also capable of performing the functions of a number of IEDs by measuring current and voltage synchrophasors. The use of SPM will significantly reduce the requirements for microcontrollers or signal processors, local medium voltage RPA devices, centralized RPA devices. The functions of centralized RPA devices can be performed by the substation input device with the implementation of busbar inherently selective protection (Fig. 1). Such protection is based on information about the synchrophasors of the current from all feeders of the medium voltage switchgear, as well as the functions of the protection of the outgoing feeders [9].

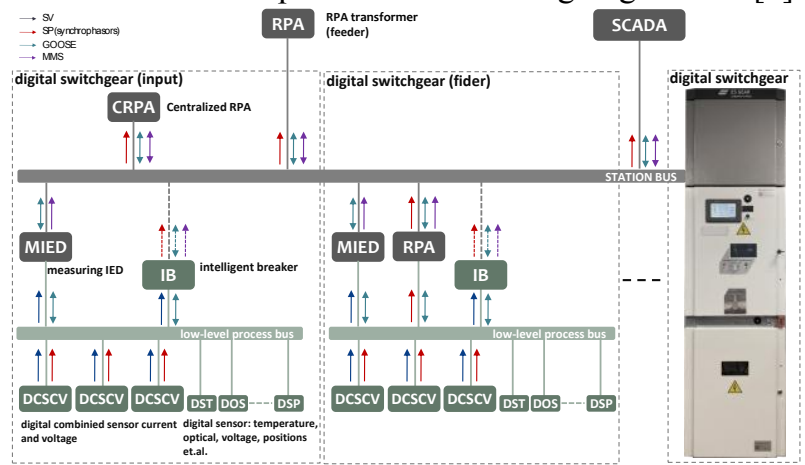

Fig. 1. Block diagram of the three-level RPA system of the stepdown substation (distribution point)

Based on the digital substation, according to Fig. 1, it becomes possible to implement a three-level distributed RPA system (smart breaker, local relay protection device, centralized RPA system), two-level protection system (smart breaker and centralized relay protection system, smart switch and local RPA device).

\section{Implementation of high-speed relay protection systems}

Current and voltage synchrophasors of a separate feeder allow to easily estimate grid mode parameters required for the implementation of current and distance protection. If there is additional information about synchrophasors from other connections, it is possible to implement differential protection of the transformer, line and bus.

The use of multi-stage overcurrent protections is often limited due to the insufficient sensitivity of individual protection stages. The sensitivity of such protections can be significantly increased by evaluating equivalent circuit parameters of the substation and power system [8]. In this case, current protection will be close in sensitivity to distance protection. But this is a topic for a separate study.

The use of SPM and fast communications in a digital substation creates an opportunity for wider use of distance and differential protection principles with a set of additional fault indicators and implementation of inherently selective protection based on the data exchange between protective relays [8]. The mass production of inexpensive microcontrollers with support for floatingpoint operations in the presence of SPM makes the application of the distance protection principle even more promising. In this case, due to the data exchange, it is also possible to implement distance inherently selective protection. With the complete degradation of the communication network, it becomes impossible to perform in inherently selective mode. Therefore, the protection relays switch to autonomous mode and with relatively selective operation. This makes it possible to reduce the requirements for the reliability of 
communications at the substation and between substations.

SPM technology allows improving line (transformer) protection and bus protection. These approaches can be extended to other connections of the substation and between substations.

In the simplest case, the operate current of the differential current protection should exceed the maximum restraint current. In the case of using traditional current transformers, the operate current must exceed $40 \%$ of the three-phase short-circuit current at the end of the line in the maximum operating mode of the power system. Assuming indicated operation threshold and the required sensitivity coefficient, the ratio of the minimum to maximum three-phase short-circuit (SC) should be more than 0.69 . This significantly limits the application of simple differential protection. The application of percentage restrained differential principle allows to increase the sensitivity of protection [10].

Another way to increase the sensitivity of differential protection is associated with the use of adaptive percentage restraint characteristics [11]. In this case, the characteristic is calculated on the basis of an estimate of the actual measurement errors of the current at the line ends and the error of time synchronization. This method reduces the above ratio between the short-circuit currents to 0.26 . Another way to increase the sensitivity of differential protection is associated with the use of a nonlinear filter of the magnetizing current [14].

In case of use of modern current sensors and current synchrophasors the more preferable method of sensitivity raising is the comparison of phases of current synchrophasors $[10,12,13]$. The use of modern current and voltage sensors in combination with the use of information about current and voltage synchrophasors in protection allows the implementation of effective inherently selective protection algorithms. The advantages of using digital current sensors for differential protection are primarily associated with their significantly better metrological characteristics (reduction of the restraint current), the absence of residual magnetization and saturation of the magnetic core at high current ratios.

The effectiveness of such inherently selective feeder protection can be demonstrated by the example shown in Fig. 2 and Fig. 3. A $6 \mathrm{kV}$ switchgear of one of the largest industrial facility in the Arkhangelsk region is presented. For simplicity, only single busbar is shown, it is from the generator voltage buses of the CHP. The busbar connections include 3 motors of $3 \mathrm{MW}$ each (only 2 shown), a 3.2 MVA transformer with a predominantly motor load, a 1 MVA transformer with a very low motor load share.

The orientation of the synchrophasors is shown relative to the incoming feeder current synchrophasor, which is normally directed towards the buses. The numbers indicate synchrophasors at the following times: 1 - normal mode, 2 - $10 \mathrm{~ms}$ after short circuit. The simulation of the power supply system was carried out using MATLAB / Simulink, taking into account the contribution of 6 and $0.4 \mathrm{kV}$ motors.

Fig. 2 shows current and voltage synchrophasors for short-circuit at the beginning, middle and end of the cable line. As follows from the presented Fig. 2, comparison of modules and phases of current synchrophasors allows the implementation of efficient inherently selective protection. In addition, to increase the protection sensitivity, the information on voltage synchrophasors of the generator bus and the switchgear busbar can be used.

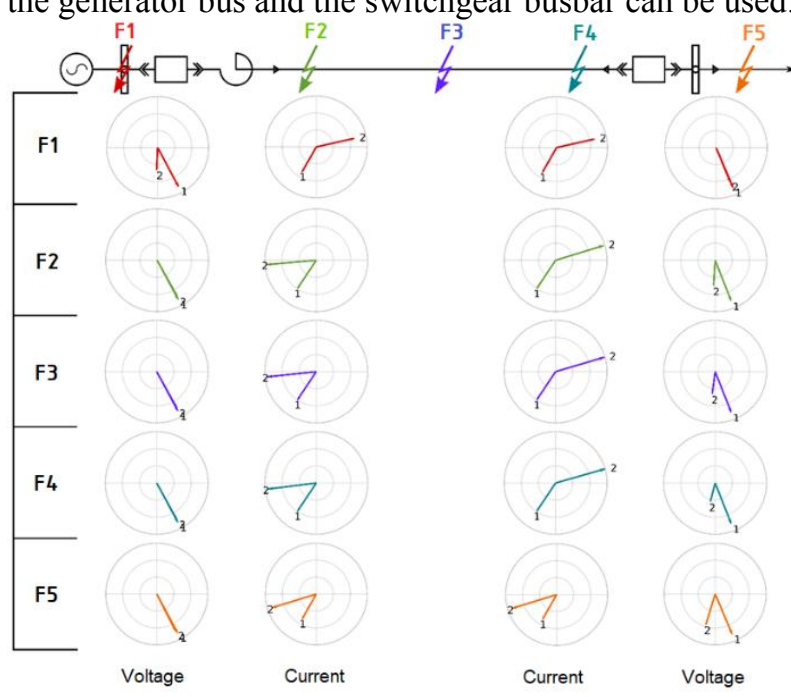

Fig. 2. Cable line protection

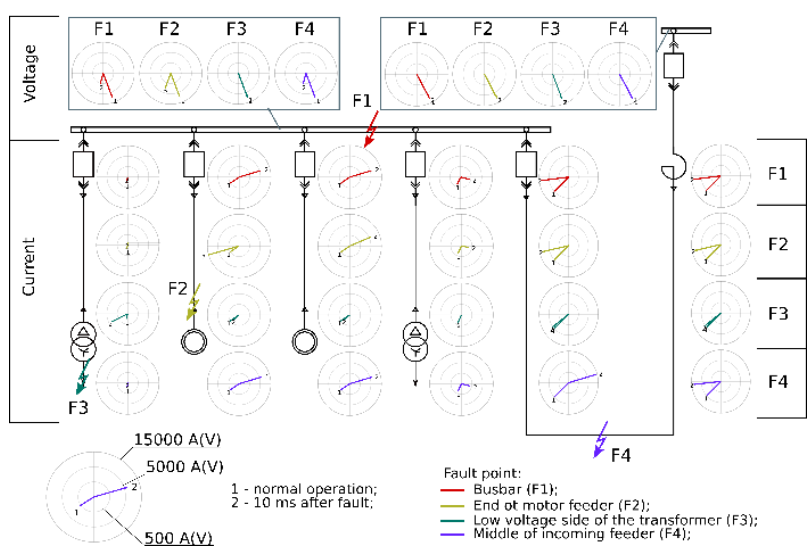

Fig. 3. Protection of busbars, cable supply lines and outgoing feeders

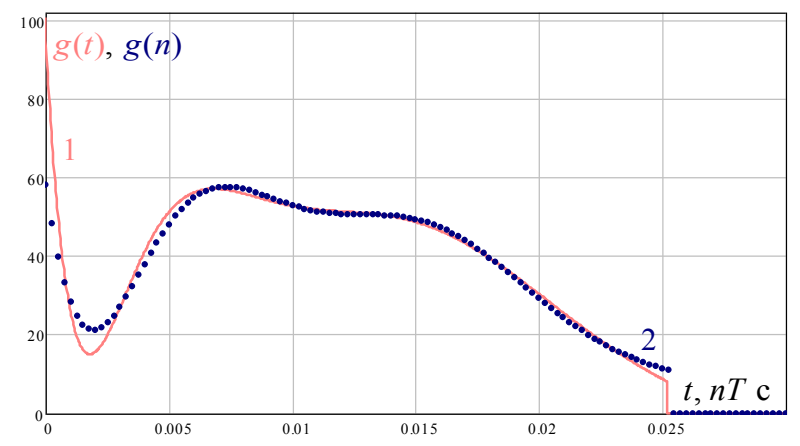

Fig. 4. Impulse functions of analog and digital filters

For the effective use of SPM in relay protection devices, it is required, as mentioned above, to use digital filters that are different from those used in PMU. For this task, an analog prototype filter (curve 1, Fig. 4) was specially synthesized using a synthesis method based on extended spectral representations of signals and using nonlinear optimization methods with limitations [15]. 
To obtain a description of the digital filter, one can use the known transition methods. But research has shown that for filters with a strongly asymmetric impulse response, it is better to use direct digital filter synthesis. Fig. 4 shows the impulse function of the digital filter under the same synthesis conditions as for filter 1 in Fig. 4 at a sampling rate of 80 points per power frequency period.
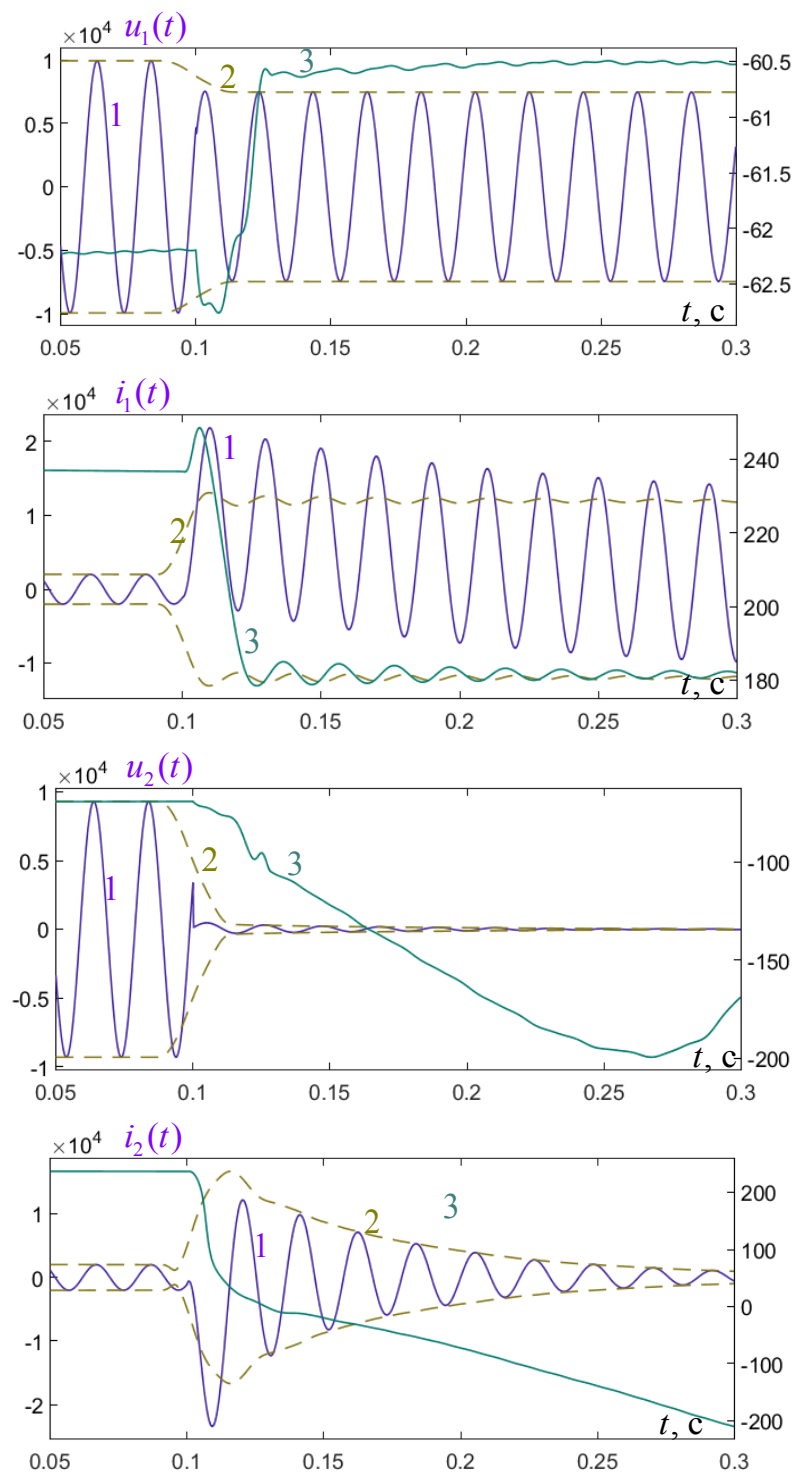

Fig. 5. Graphs of currents, voltages, modules and arguments of synchrophasors

Figure 5 shows the graphs of voltage and current at the beginning $\left(\mathrm{u}_{1}(\mathrm{t}), \mathrm{i}_{1}(\mathrm{t})\right)$ and at the end of the line $\left(\mathrm{u}_{2}(\mathrm{t}), \mathrm{i}_{2}(\mathrm{t})\right)$, as well as graphs of the corresponding voltages and currents (curves 1) of the modules (curves 2) and arguments (curves 3 ) of synchrophasors in case of a short circuit in the middle of the protected line. For illustration, the current envelope at the end of the line, determined on the basis of the current synchrophasor module, is shifted to the left along the time axis by a value equal to the group delay of the filter.

Studies have shown that stable operation of the cable line protection is provided with a large margin after $10 \mathrm{~ms}$ after the short circuit. In this case, the duration of the impulse function of the filter is $27 \mathrm{~ms}$ (Fig. 4).

In the work [8] it is shown that by using synchrophasors it is possible to implement bus inherently selective protection, combining the differential principle with the control of arguments (phases) and modules of feeder current synchrophasors (Fig. 3). In the bus differential protection, only the difference between the incoming and sum of outgoing currents is used. Therefore, the use of additional information about the amplitudes and phases of the incoming currents based on the SPM allows increasing the sensitivity and speed of protection.

\section{Estimation of current transformer saturation effect}

The examples of application of inherently selective protection based on synchrophasors shown above were considered for the case when modern current sensors are used, in which saturation and residual magnetization errors are absent or minimized.

In cases where traditional current transformers are used, it is proposed to use an algorithm based on the restoration of the equivalent fundamental harmonic current synchophasor based on the information on the higher harmonic synchrophasors.

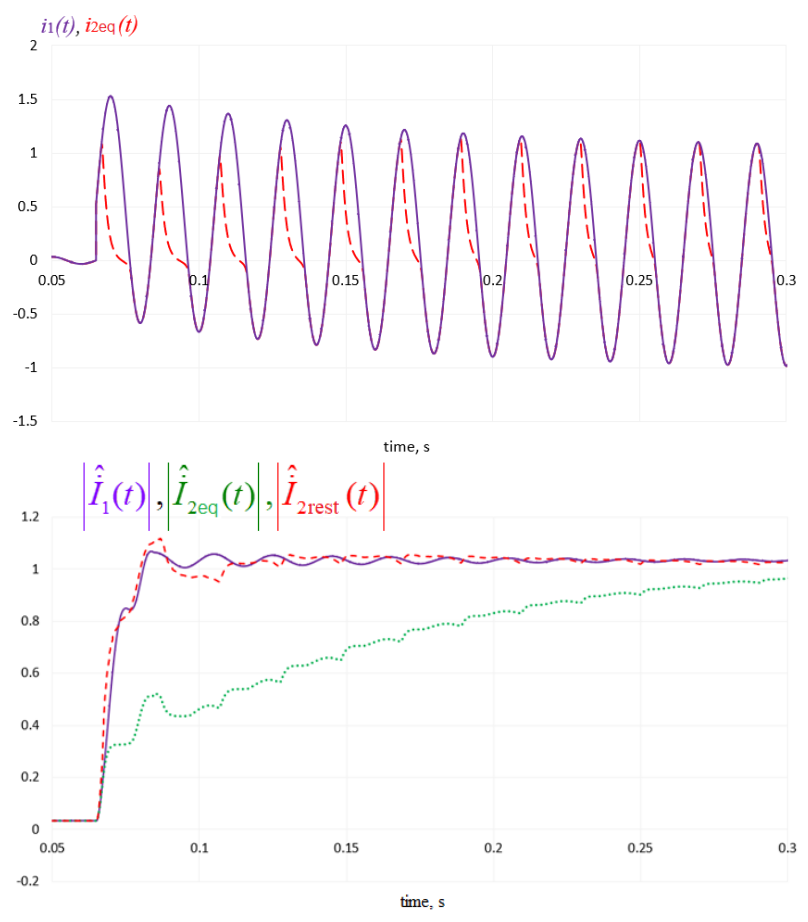

Fig. 6. Restoration of the synchrophasor module of the equivalent secondary current

Fig. 6 shows an example of such a restoration. The calculation of the value of the restored current is carried out according to a predetermined relationship between the degree of saturation of the current transformer and the relative magnitude of the harmonics. This characteristic is similar for current transformers of the same type, which greatly simplifies the restoration algorithm and allows use this dependence for current transformers with different rated currents. 


\section{Conclusions}

The prospect of using the SPM technology for relay protection of step-down substations and distribution points is associated with the possibility of widespread introduction of high-speed inherently selective protections, as well as increasing the functionality of the protections. The design of local and distributed relay protection systems with the support of SPM technology implies that special attention should be paid to the modeling of the protected object and protection, and the synthesis of high-speed algorithms for relay protection and automation.

\section{References}

1. H.J.A. Ferrer, E.O. Schweitzer III (eds.), Modern Solutions for Protection, Control, and Monitoring of Electric Power Systems(SEL,Pullman, 2010).

2. E.M. Schneerson, T. Liebach, Relay Protection and Automation of Power Systems (2004).

3. Yu. Ya. Lyamets, G.S. Nudelman, D.V. Kerzhaev, $\mathrm{Yu}$.V. Romanov, Modern trends in the development of relay protection systems and automation of power systems (2009).

4. I.V. Nagai, Relay protection and automation of power systems (2010).

5. E.A. Emerson, Handbook of Theoretical Computer Science (1990).

6. M. Zeng-Qiang, Z. Hong-Shan, W. Hai-Ping, L. Ying, IEEE/PES Transmission \& Distribution Conference(2005).

7. A.V. Mokeev et al., Operational management in the electric power industry, 1 (2016).

8. A.V. Mokeev, E.I. Khromtsov, Relay protection and automation, 4, (2019).

9. A.V. Bulychev et al. Relay protection and automation, 1, (2019).

10. A.M. Fedoseev Relay protection of electric power systems (EAI, 1992).

11. G. Ziegler Digital devices of differential protection. Principles and scope (EI, 2005).

12. S.L. Kuzhekov, V. Ya. Sinelnikov, Busbar Protection for Power Plants and Substations (EAI, 1983).

13. E.I. Bass, V.G. Doroguntsev, Relay Protection of Electric Power Systems (MEI, 2002).

14. V.K. Vanin, M.G. Popov, S.O. Popov, Scientific and Technical Sheets of St. Petersburg State Pedagogical University, 3 (2011).

15. A.V.Mokeev, IEEE International Conference SIBCON-2017 (2017). 